\title{
A rare presentation of hypertrophic cardiomyopathy in a neonate
}

\author{
T M R Gunasekera ${ }^{1}$, D K Samarage ${ }^{2}$, M V C de Silva ${ }^{3}$, W D N Weerapperuma ${ }^{4}$ \\ Sri Lanka Journal of Child Health, 2007; 36: 114-115
}

(Key words: hypertrophic cardiomyopathy, neonate)

\section{Introduction}

Hypertrophic obstructive cardiomyopathy (HOCM) is reported to have an incidence of 2.5 per 100,000 population, accounting for $20-30 \%$ of all cases of paediatric primary myocardial disease ${ }^{1}$. HOCM is inherited primarily in an autosomal dominant manner with incomplete penetrance ${ }^{2}$, or as a maternally derived mitochondrial disorder ${ }^{3}$. It also occurs as a secondary phenomenon in genetic syndromes (Noonan syndrome), metabolic disorders (Pompe mucopolysachcharidosis), and endocrine disorders (hypothyroidism). HOCM can occur as a transient phenomenon in Beckwith-Widemann syndrome, in infants of diabetic mothers or in pre-term infants treated with steroids for chronic lung disease. Mutations of the cardiac beta myosin heavy chain gene on chromosome 14 are responsible for $30-40 \%$ of familial cases ${ }^{4}$. Certain mutations are responsible for good clinical outcome, whereas others result in early death ${ }^{5}$. There are only a few reported cases of HOCM in the neonatal period ${ }^{6,7,8}$.

\section{Case History}

A nine day old female neonate was admitted to hospital with a six hour history of irritability and poor sucking. She was the second child born to healthy non-consanguineous parents and the elder sibling, a six year old male remains healthy. The antenatal period was not complicated by diabetes and the birth was uneventful with a birth weight of $2.8 \mathrm{~kg}$. There was no family history of heart disease or any unexplained sudden deaths. Examination revealed the neonate to be in total circulatory collapse with cardiomegaly and no heart murmurs. A $4 \mathrm{~cm}$ hepatomegaly was found on abdominal examination. There were no dysmorphic features noted.

${ }^{1}$ Registrar, Colombo South Teaching Hospital, ${ }^{2}$ Head of Department \& Senior Lecturer, Department of Paediatrics, Faculty of Medical Sciences, University of Sri Jayawardanapura. ${ }^{3}$ Professor of Pathology, Faculty of Medicine, Colombo. ${ }^{4}$ Demonstrator, Department of Paediatrics, Faculty of Medical Sciences, University of Sri Jayawardanapura

(Received on 2 October, 2006)
The neonate was resuscitated and immediately transferred to the intensive care unit. The basic hematological investigations did not reveal any abnormality and there were no laboratory evidence of sepsis. Chest x-ray revealed gross cardiomegaly. Left ventricular hypertrophy was evident on ECG. 2D echocardiography demonstrated gross cardiomegaly with hypertrophy of the left ventricle and the interventricular septum. The ejection fraction was $20 \%$.

The neonate was ventilated and treated with antifailure drugs including captopril, diuretics and intravenous antibiotics. The neonate's condition deteriorated while on the ventilator and the baby expired on day 31 of life.

\section{Pathological findings}

The heart was enlarged and globular shaped. It was in atrial situs solitus with atrio-ventricular and ventricular-arterial concordance. The left ventricle was hypertrophied and the interventricular septum was markedly thickened and was protruding into left ventricular cavity. There was marked narrowing of the aortic orifice. Histologically, cardiac muscle fibres showed mild nuclear enlargement with hypertrophy.

\section{Discussion}

HOCM is a heterogeneous clinical disorder with a myriad of morphological, clinical and pathophysiological features. It has a characteristic cardiac involvement with a hypertrophic, non-dilated left ventricle. HOCM can present at any age though neo-natal presentations are extremely rare. Many are asymptomatic. Weakness, easy fatiguability, dyspnoea on effort, palpitations, angina and dizziness are main symptoms in adults and older children. There is a risk of $1-2 \%$ per year of sudden death even in asymptomatic. $50 \%$ of HOCM patients are diagnosed during screening due to an affected family member or due to detection of a cardiac murmur. ECG revealing left ventricular hypertrophy without ST depression and $\mathrm{T}$ inversions, roentegenograhy demonstrating cardiomegaly with left ventricular 
prominance, echocardiography showing left ventricular hypertrophy with predominant interventricular septum and Doppler studies revealing left ventricular outflow tract gradient are the main diagnostic tools. Cardiac catheterization assesses the suitability for surgery.

The prognosis and the disease course are unpredictable. The main complications are congestive cardiac failure and sudden death from cardiac arrhythmias. The management strategies include life style modifications with avoidance of strenuous physical activity and competitive sports in older patients. The drug treatment options include $\beta$ adrenergic blockers and calcium channel blockers which will reduce the development of cardiac hypertrophy. Arrhythmias require cardiac pacing. The ventricular septal myomectomy is reserved for those with severe drug refractory symptoms.

\section{References}

1. Codd M B, Sugrue D D, Gersh B J, et al. Epidemiology of idiopathic dilated and hypertrophic cardiomyopathy Circulation.1991; 83:515.

2. Maron B J, Nicholos P F, Pickle L W, et al. Patterns of inheritance in hypertrophic cardiomyopathy: Assessment by M-mode and two-dimensional echocardiography. $\mathrm{Am} J$ Cardiol 1984; 53: 1087.
3. Bione S D, Adamo P, Maestrini E, et al. A novel $\mathrm{X}$-linked gene, G4.5 is responsible for Barth syndrome. Nat Genet 1996; 12: 385.

4. Behrman R E, Kliegman R M, Jenson H B. Editors, Nelson Text book of Paediatrics. $17^{\text {th }} \mathrm{ed}$. Philadelphia: Saunders; 2004. p.1575-6.

5. Watkins H, Rosenzweig A, Wang DS, et al. Characteristics and prognostic implications of Myosin missense mutations in familial hypertrophic cardiomyopathy. New England Journal Medicine.1992; 326:1108.

6. Eidem B W, Jones C. Unusual associations of Hypertrophic Cardiomyopathy with complete atrio-ventricular canal defect and Down syndrome. Texas Heart Journal .2000; 27(3):289-91.

7. Smith W Q, Abu_H, Harb M. Undiagnosed cardiomyopathy in a neonate. British Journal of Anaesthesia. 2001; 86(3):435-7.

8. Phadke R S, Vaideeswar P, Mittal B, Deshpande J. Hypertrophic cardiomyopathy: an autopsy analysis of 14 cases. Journal of Post-graduate Medicine.2001; 47(3):165-70. 